\title{
Skeletal muscle phenotyping of Hippo gene-mutated mice reveals that Lats 1 deletion increases the percentage of type I muscle fibers
}

\author{
Fakhreddin Yaghoob Nezhad (D) Annett Riermeier • Martin Schönfelder • \\ Lore Becker (1) - Martin Hrabě de Angelis (D) - Henning Wackerhage (i)
}

Received: 24 May 2021/Accepted: 2 December 2021/Published online: 5 January 2022

(C) The Author(s) 2022

\begin{abstract}
The Hippo signal transduction network regulates transcription through Yap/Taz-Tead1-4 in many tissues including skeletal muscle. Whilst transgenic mice have been generated for many Hippo genes, the resultant skeletal muscle phenotypes were not always characterized. Here, we aimed to phenotype the hindlimb muscles of Hippo gene-mutated Lats $^{-1-}$, Mst $^{-1-}, \mathrm{Vgll}^{-/-}$, and $\mathrm{Vgll}^{+/-}$mice. This analysis revealed that Lats $^{-/-}$mice have $11 \%$ more slow type I fibers than age and sex-matched wild-type controls. Moreover, the mRNA expression of slow
\end{abstract}

Supplementary Information The online version contains supplementary material available at https://doi.org/10.1007/ s11248-021-00293-4.

F. Y. Nezhad · A. Riermeier · M. Schönfelder ·

H. Wackerhage $(\square)$

Exercise Biology Group, Faculty of Sport and Health

Sciences, Technical University of Munich, Munich,

Germany

e-mail: henning.wackerhage@tum.de

L. Becker - M. H. de Angelis

German Mouse Clinic, Institute of Experimental Genetics,

Helmholtz Zentrum München, Neuherberg, Germany

M. H. de Angelis

Chair of Experimental Genetics, TUM School of Life

Sciences, Technische Universität München, Freising,

Germany

M. H. de Angelis

German Center for Diabetes Research (DZD),

Neuherberg, Germany
Myh7 increased by $50 \%$, and the concentration of type I myosin heavy chain is $80 \%$ higher in Lats $^{-1-}$ mice than in age and sex-matched wild-type controls. Second, to find out whether exercise-related stimuli affect Lats1, we stimulated C2C12 myotubes with the hypertrophy agent clenbuterol or the energy stress agent AICAR. We found that both stimulated Lats1 expression by 1.2 and 1.3 fold respectively. Third, we re-analyzed published datasets and found that Lats 1 mRNA in muscle is $63 \%$ higher in muscular dystrophy, increases by $17-77 \%$ after cardiotoxin-induced muscle injury, by $41-71 \%$ in muscles during overloadinduced hypertrophy, and by $19-21 \%$ after endurance exercise when compared to respective controls. To conclude, Lats1 contributes to the regulation of muscle fiber type proportions, and its expression is regulated by physiological and pathological situations in skeletal muscle.

Keywords Hippo pathway - Skeletal muscle · Transgenic mice $\cdot$ Lats1 Fiber type

\section{Introduction}

We use our $\approx 650$ human skeletal muscles to move, stand, speak, generate heat, store glucose as glycogen, and amino acids as protein. Skeletal muscles also vary greatly within and in-between individuals. For 
example, muscle contributes from 20 to $50 \%$ to total human body mass (Janssen et al. 2000) and this is due to a more than twofold variation of mean fiber crosssectional areas and fiber numbers (Lexell et al. 1988). In addition, genetics and years of training can increase muscle mass and function in athletes (Sarzynski and Bouchard 2020) whereas aging, disability, immobilization, and diseases such as muscular dystrophy, cancer, and diabetes impair or reduce muscle metabolism, function, and size (Bonaldo and Sandri 2013; Wolfe 2006).

Muscle size and function are regulated by many signaling pathways and depend on genetics. In the past two decades, the Hippo signal transduction network has been identified as a regulator of skeletal muscle development, regeneration, and size and is associated with diseases such as muscular dystrophy and rhabdomyosarcoma (Wackerhage et al. 2014). In mammals, the canonical Hippo pathway consists primarily of the kinases MST1, MST2, LATS1, and LATS2 (Gabriel et al. 2016). Activated LATS1/2 inhibits the transcriptional co-factors YAP/TAZ by phosphorylation of multiple serines (Liang et al. 2014; Zhao et al. 2007). In contrast, unphosphorylated YAP/ TAZ can translocate to the nucleus to co-activate TEAD-1-4 transcription factors to initiate the transcription of target genes such as ANKRD1,CTGF, and CYR61 (Dong et al. 2007; Liu et al. 2010; Oh and Irvine 2008; Ren et al. 2010; Zhao et al. 2010). Finally, VGLL1-4 proteins can also bind TEAD1-4 (Zhou et al. 2016) and may compete for common binding interfaces with Yap and Taz (Figeac et al. 2019; Hori et al. 2020; Koontz et al. 2013; Yamaguchi 2020).

Hippo researchers and the International Mouse Phenotyping Consortium (IMPC; https://www. mousephenotype.org/) have generated Hippo mutant mice for almost all Hippo-related genes. These mice have all been generally phenotyped but a specific, quantitative phenotyping of skeletal muscle is rarely performed and often muscles are not phenotyped at all.

The aim of this study was therefore to phenotype hindlimb muscles of Lats $1^{-1-}$, Mst $^{-/-}$, Vgll3 $^{-/-}$, and $\mathrm{Vgll}^{+/-}$mutated mice and to compare these transgenic mice to age and sex-matched wild-type controls.

\section{Materials and methods}

Ethical approval and husbandry

All animal procedures including ethical statements and housing $\&$ husbandry conditions are available on the IMPC portal website (http://www. mousephenotype.org/about-impc/arrive-guidelines).

Mouse generation and phenotyping

To study the role of the Hippo related genes in skeletal muscle, the hindlimbs of global knockout mice include Lats $1^{-/-} \quad\left(\right.$ Lats $\left.I^{\text {em1(IMPC)H}}\right), \quad M s t 2^{-1-}$ $\left(\right.$ Stk $\left.3^{\mathrm{em} 1(\mathrm{IMPC}) \mathrm{H}}\right), \quad V g l l 3^{-/-}\left(\right.$Vgll $\left.^{\mathrm{em} 1(\mathrm{IMPC}) \mathrm{H}}\right)$, and $\mathrm{Vgll}^{+/-}\left(\mathrm{Vgll4}^{\mathrm{tm} 1 \mathrm{~b}(\mathrm{EUCOMM}) \mathrm{Hmgu}}\right)$ were kindly supplied by the Helmholtz Zentrum München (HMGU) in Germany, and the Medical Research Council Harwell (MRC Harwell) in the UK. Mice were generated on a C57BL/6 $\mathrm{N}$ background and phenotyping data were collected at the age of 16 weeks. For each of the Hippo-related genes knockout lines, at least three hind limbs of male mice were collected as well as age and sex-matched control mice of equivalent genetic backgrounds and sent to the Technical University of Munich for hindlimb muscle phenotyping. Information on the transgenesis, phenotyping, and data storage is available on the IMPC portal:

https://www.mousephenotype.org/understand/thedata/allele-design/

https://www.mousephenotype.org/understand/thedata/phenotyping-process\%20impress/

\section{http://www.mousephenotype.org/}

To phenotype the hindlimb muscles of Hippo genemutated mice and their matched control, we first dissected the hindlimb muscles of mutated and control mice. The analyzed muscles are the tibialis anterior (TA), extensor digitorum longus (EDL), gastrocnemius, and soleus. After dissection, we weighed the hindlimb muscles and then calculated the relative muscle weight (muscle weight/whole body weight). 
Mouse genotyping

Each Lats $^{-/-}$and wild-type control mouse was genotyped using mouse skeletal muscle DNA. To isolate DNA, the EDL muscles were digested in lysis buffer ( $350 \mu \mathrm{l}$ of Sodium hydroxide (NaOH $50 \mathrm{mM})$, and $10 \mu \mathrm{l}$ proteinase $\mathrm{K}$ per sample). Following overnight incubation at $55^{\circ} \mathrm{C}, 120 \mu \mathrm{l}$ of $5 \mathrm{M} \mathrm{NaCl}$ was added and centrifuged for $15 \mathrm{~min}$, and resulting supernatant $(350 \mu \mathrm{l})$ was added to $250 \mu \mathrm{l}$ of isopropanol (Carl Roth, Germany). After vortexing for $2 \mathrm{~min}$, centrifugation at $13,000 \mathrm{rpm}$ for $10 \mathrm{~min}$, and $2 \mathrm{~h}$ incubation at room temperature (RT), the aqueous phase was collected. DNA precipitation was obtained by addition of $500 \mu \mathrm{l}$ of $70 \%$ Ethanol, short vortexing, and $10 \mathrm{~min}$ centrifugation at $13,000 \mathrm{rpm}$ at $4{ }^{\circ} \mathrm{C}$. Finally, the DNA pellets were resuspended in $50 \mu \mathrm{l}$ $\mathrm{ddH} 2 \mathrm{O}$ and were quantitated on a Nanodrop spectrophotometer (ND-100 spectrophotometer; Nanodrop Technologies, Wilmington, DE, USA). For this genotyping study, DNA was amplified using a TAQ-DNAPolymerase kit (PeqGold VWR, product number 01-1020) as specified by the manufacturer by a PCR master cycler (Eppendorf, Germany). The amplification cycles consisted of $4 \mathrm{~min}$ at $95{ }^{\circ} \mathrm{C}$ followed by 30 cycles at $95{ }^{\circ} \mathrm{C}$ for $30 \mathrm{~s}, 58{ }^{\circ} \mathrm{C}$ for $30 \mathrm{~s}$, and $72{ }^{\circ} \mathrm{C}$ for $2.5 \mathrm{~min}$. The primer sequences were as follows: Lats1: forward (F) 5' - TCCGGGCAGGACTGATATAC -3'; reverse (R) 5' $5^{\prime}$ TATTACAGGAAATGCTGAATAACTG $-3^{\prime}$. The amplification of Lats 1 knock-out and wild-type gene fragments results in 202-bp and 2071-bp products, respectively. An agarose gel electrophoresis of the PCR products is shown in Fig. S3 (see supplementary data).

\section{Cell culture}

Mouse C2C12 myoblasts cells were cultured in Dulbecco's modified Eagle's medium (DMEM) (Gibco, Cat\#31,885, Waltham, MA, USA), supplemented with $10 \%$ fetal bovine serum (Sigma, Germany), and incubated at $37{ }^{\circ} \mathrm{C}$ in humidified air with $5 \% \mathrm{CO}_{2}$. To induce differentiation, the myoblasts were cultured in a growth medium until confluence, then the medium was switched to DMEM with $2 \%$ horse serum (Sigma, Germany). The proliferation and differentiation medium was refreshed daily up to $96 \mathrm{~h}$. After $48-96 \mathrm{~h}$ of differentiation, C2C12 Myotubes untreated (DMSO) or $24 \mathrm{~h}$ treated with exercise stimulates including $100 \mu \mathrm{M}$ Clenbuterol (Sigma, Germany) to induce hypertrophy, or $1 \mathrm{mM}$ 5-aminoimidazole-4-carboxamide-1- $\beta$-D-ribofuranoside (AICAR; Cell Signaling Technology, USA) to induce energy stress.

RNA isolation, reverse transcription, and quantitative real-time PCR

Following dissection, the soleus muscle samples were homogenized in $1 \mathrm{ml}$ of QIAzol (QIAGEN, Germany) using a Precellys lysing kit (Bertin Instruments, USA) and Precellys homogenizer machine (Bertin Technologies, USA) with high speed for $40 \mathrm{~s}$. C2C12 myotubes were first washed with phosphate-buffered saline (PBS) and then lysed in lysis T buffer (Peqlab Biotechnology GmbH, reference number 12-6634-01) and stored at $-80 \mathrm{C}$. Total RNA was extracted with the peqGOLD Total RNA Kit C-Line (Peqlab Biotechnology $\mathrm{GmbH}$, reference number: 12-6634-01) according to the manufacturer's instructions. RNA concentrations were determined by a Nanodrop spectrophotometer (ND-100 spectrophotometer; Nanodrop Technologies, Wilmington, DE, USA). RNA purity was ensured by a $260 / 280$ ratio (range 2.00-2.11, mean 2.04). For mRNA analysis, cDNA was synthesized using $2 \mu \mathrm{g}$ of total RNA and qScript XLT cDNA SuperMix (Quantabio, product number 030256) as specified by the manufacturer. cDNA was amplified with a PerfeCTa qPCR fluorescent SYBR Green SuperMix (Quantabio, product number: 023916) using real-time quantitative PCR (Rotor-Gene RG 6000QIAGEN). The primer sequences used for the gene expression were as follows:

Lats 1: forward (F) $5^{\prime}-$ AATGAAATGATGCGGGTTGGA-3'; reverse (R) 5'- CAGACTTCACCAAACGCTCC- $3^{\prime}$;

Rpl7: (F) 5'-ACGGTGGAGCCTTATGTGAC-3' and (R) $5^{\prime}$-TCCGTCAGAGGGACTGTCTT- ${ }^{\prime}$.

We calculated the expression of Lats 1 using the $2^{-\Delta \Delta \mathrm{Ct}}$ method (Livak and Schmittgen 2001) and calculated it relative to the $R p l 7$ housekeeping gene (Thomas et al. 2014).

Western blotting

To extract total protein, we homogenized soleus muscles in lysis buffer $(0.1 \%$ SDS, $0,5 \mathrm{M}$ sodium orthovanadate, $0.5 \%$ sodium deoxycholate, $50 \mathrm{mM}$ 
$\mathrm{NaF}, 1 \mathrm{mM}$ EDTA, $150 \mathrm{mM} \mathrm{NaCl}, 1 \%$ Triton-X 100) supplemented with protease and phosphatase inhibitor cocktail (Peqlab Biotechnology GmbH, Germany) using a Precellys lysing kit (Bertin Instruments, USA) and a homogenizer machine (Bertin Technologies, USA) with high speed for $40 \mathrm{~s}$. After centrifugation at $12,000 \mathrm{~g}$ for $10 \mathrm{~min}$ at $4{ }^{\circ} \mathrm{C}$, the supernatant was transferred to a new tube and assayed to determine the protein concentration of each sample using the Bradford protein assay kit (BioRad Laboratories $\mathrm{GmbH}$, Cat \#5,000,111, Munich, Germany). Homogenates of each soleus sample were diluted to a protein concentration of $1 \mathrm{mg} / \mathrm{ml}$ using $4 \times$ SDS sample buffer (0.5 M Tris- $\mathrm{HCl} \mathrm{pH} 6.8,10 \%$ glycerol, $2 \%$ sodium dodecyl sulfate, $20 \% \beta$-mercaptoethanol, and $0.05 \%$ bromophenol blue). Samples were then heated at $95 \mathrm{C}$ for $5 \mathrm{~min}$, and $30 \mu \mathrm{g}$ of whole protein lysates were separated by electrophoresis in a $10 \%$ SDS-PAGE gel (Bio-rad, Germany). Proteins were then transferred to polyvinylidene difluoride (PVDF) membranes (Biorad, Germany) using a Trans-Blot Turbo Blotting System (Bio-Rad, Germany). Following a blocking step (5\% non-fat milk powder, 1X Tris-buffered saline, $1 \%$ Tween-20), the PVDF membrane was incubated overnight with primary antibody and probed with horseradish peroxidase(HRP)-linked secondary antibodies for $1 \mathrm{~h}$ at room temperature. The membranes were developed with enhanced chemiluminescence (ECL) (Bio-Rad, Germany), and the signals were detected by an INTAS Chemocam Imager (Royal Biotech $\mathrm{GmbH}$, Germany). The antibodies used in this study were as follows: Anti MyHC-1 (1:1000, DSHB Cat\# BA-D5, RRID: AB_2235587), Anti-mouse IgG, HRP-linked Antibody (1:2500, Cell Signaling Technology Cat\# 7076, RRID: AB_330924). Quantification of immunoreactive bands was performed using the ImageJ software (http://rsb.info.nih.gov/ij/index.html) and the largest band of a Ponceau S stain of the membrane was used for normalization (Romero-Calvo et al. 2010).

Muscle cryosectioning and histology

The tibialis anterior (TA) and soleus muscles of mice were dissected, frozen in isopentane (2-methyl butane) cooled to near freezing with liquid nitrogen, and sectioned in a cryostat (LEICA CM3050 S) and mounted on SuperFrost ${ }^{\circledR}$ slides (VWR International
GmbH, Germany Cat\#631-1349) air-dried and stored at $-80 \mathrm{C}$ until further analysis.

Haematoxylin \& eosin stain \& NADH tetrazolium reductase histochemical stain

To find out whether the mutation of Hippo genes causes abnormalities such as central nuclei, infiltrations or fibrosis, we stained all muscles with hematoxylin and eosin (H\&E) using a standard staining protocol. To stain highly oxidative (typically corresponding to type I and IIa fibers) and less oxidative fibers (corresponding to type IIx and IIb fibers), we used an NADH tetrazolium reductase (NADH-TR) histochemical reaction. For this, frozen TA muscle cryosections $(10 \mu \mathrm{m})$ were allow to thaw at room temperature and incubated with NADH-TR staining solution $(2.52 \mathrm{~g}$ Tris $\mathrm{HCl}, 0.10 \mathrm{~g}$ Nitro blue tetrazolium, $0.68 \mathrm{~g}$ Tris base, dissolved in $100 \mathrm{ml}$ distilled $\mathrm{H}_{2} \mathrm{O} \& \approx 1 \mathrm{mg}$ of $\mathrm{NADH}$ added to $1 \mathrm{ml}$ staining solution ( $\mathrm{pH} 7.4$ ) before staining) for 30-60 min in a humidified chamber at room temperature until there was maximal contrast in staining intensity in-between fibers. Then, samples were washed in distilled water.

Adenosine triphosphatase (ATPase) histochemical stain

To differentiate in-between type I and type II muscle fibers we used an ATPase histochemical reaction with acid preincubation to selectively stain type I fibers brown-black. For this, $10 \mu \mathrm{m}$ soleus sections were thawed at room temperature for $5 \mathrm{~min}$ and preincubated in an acetate buffer at $\mathrm{pH} 4.47$ for $10 \mathrm{~min}$ followed by an Adenosine 5'-triphosphate reaction solution for $30 \mathrm{~min}$ at $37 \mathrm{C}$ then rinsing in distilled water. The slides were then incubated in $1 \%$ calcium chloride for $3 \mathrm{~min}$ then rinsed in distilled water and placed in $2 \%$ cobalt chloride solution for $3 \mathrm{~min}$. Following further washes in distilled water, slides were finally incubated in a $1 \%$ ammonium sulfide solution for $1 \mathrm{~min}$ and washed 3 times in distilled water.

\section{Dehydration and Mounting}

All sections from H\&E staining, NADH-TR, and ATPase enzyme activity analysis were dehydrated by sequential dipping in $70 \%$ and $100 \%$ ethanol and then 
ROTI Histol (Carl Roth, Germany), before being mounted with coverslips using DPX mounting medium.

\section{Microscopy}

Digital photos of cross-sections were captured in 2.5, 5, and 20-X magnifications using a Zeiss Axio Lab.A1 equipped with a digital camera (Zeiss, Germany) and Zeiss ZEN Software version 2.6 (Blue edition; Zeiss, Germany). To determine the cross-sectional area (CSA), Twenty-five myofibers per fiber type, per sample were analyzed. To distinguish fiber types (type I and II) percentages of muscle fiber types were quantified manually by counting stained and unstained fibers (fivefold magnification). All microscopy analysis was performed blinded using the ImageJ software (http://rsb.info.nih.gov/ij/index.html;RRID:

SCR_003070).

Re-analyses of published datasets

To investigate whether diseases, exercise, or other factors affect Lats1 expression we retrieved gene expression datasets from Gene Expression Omnibus (GEO). These include dataset (GDS4924) for the regeneration after cardiotoxin-induced muscle injury in TA muscle of mice (Lukjanenko et al. 2013), the transcriptome dataset (GSE23244) for comparison fiber type I and llb in mouse muscle (Chemello et al. 2011), microarray dataset GDS4932 for muscle hypertrophy after synergist ablation-overloaded in plantaris muscle of mice (Chaillou et al. 2013), and microarray dataset (GDS609) for muscle dystrophy in quadriceps muscle of Duchenne Muscular Dystrophy (DMD) patients and unaffected controls (see the supplementary data S1A in Haslett et al. (Haslett et al. 2003). To obtain data on the effects of exercise on Lats1 expression, we downloaded supplementary data files from studies comparing resting muscle biopsies with biopsies taken at $2.5 \mathrm{~h}$ and $5 \mathrm{~h}$ after a bout of either endurance or resistance (strength) exercise (Vissing and Schjerling 2014). Lastly, to obtain a forest plot of the expression of Lats1 gene in response to acute aerobic and acute resistance exercise we re-analyzed the meta-analysis of all available human skeletal muscle studies. The original data was obtained from Meta MEx Portal (http://www. metamex.eu/) (Pillon et al. 2020).
Statistical analysis

To analyze the normal distribution of data we performed a Kolmogorov-Smirnov (K-S) normality test and then performed an unpaired t-test to test for differences between conditions. Results are expressed as mean \pm standard error (SEM) and considered significant at $P<0.05$. All statistical analyses were performed using Prism version 8.0 statistical software package (GraphPad Prism; RRID: SCR_002798).

\section{Results}

Effect of Hippo gene mutations on the absolute and relative size of hindlimb muscles

To gain information on general phenotypes of Hippo transgenic mice, we first summarised publicly available IMPC phenotype data in Supplemental Table S1. Key phenotypes of Hippo gene-mutated mice were a $52 \%\left(P<1.91 \times 10^{-06}\right)$ higher fat mass in Teadl ${ }^{1-}$ female mice, a $33 \%\left(P=1.64 \times 10^{-06}\right)$ higher grip strength in Lats $^{-1-}$ female mice, and $18.6 \%(P=$ $\left.1.69 \times 10^{-08}\right)$ less lean body mass in Lats $^{-1-}$ male mice (Fig. 1a). Moreover grip strength was $27 \%$ $\left(P=2.92 \times 10^{-06}\right)$ lower in Lats $1^{1-}$ male mice when compared to sex- and age-matched wild-type mice (Supplemental table. S1).

Next, to study the effect of transgenesis on skeletal muscle phenotype, we phenotyped hindlimb muscles of $\mathrm{Lats}^{-/-}, \mathrm{Mst}^{-/-}, \mathrm{Vgll} \mathrm{3}^{-/-}$, and $\mathrm{Vgll}^{+/-}$mice versus sex- and age-matched wild-type mice as controls. The hindlimb of these mice were available from our collaborators (see Acknowledgments). Lat$s 1^{-/-}$muscles were significantly lighter than those of wild-type controls (Fig. 1b) but this is explained by a proportionally lower body weight so that relative muscle weight was unchanged (Fig. 1c). The lower body weight of $\mathrm{Lats}^{-1-}$ mice is probably explained by the lower growth hormone levels reported in an earlier study of Lats 1 transgenic mice (St John et al. 1999). When inspecting H\&E stains of all muscles, we did not detect pathological changes such as central nuclei or cores in any of the mutant mice (Fig. 1d, e; Fig. S1). 


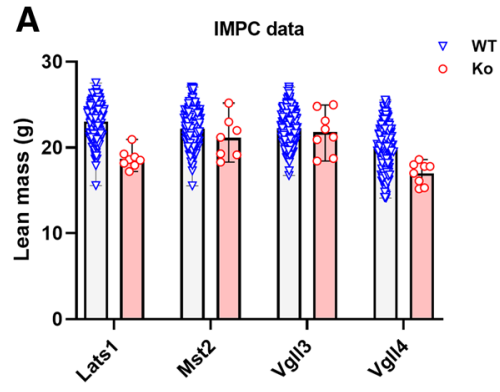

D

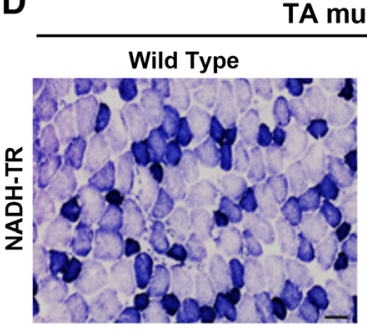

Fig. 1 (a) Lean mass of Hippo gene-mutated $(n=6-8)$, and control ( $n=499-1850$ ) 16-week-old mice. (b) Average of total body weight of Lats1 knockout $(\mathrm{n}=8)$ and its control ( $\mathrm{n}=3349$ ) mice. (c) Relative muscle weights of Gastrocnemius (Gas), Tibialis Anterior (TA), Extensor Digitorum Longus (EDL), and Soleus (Sol) muscles normalized to total body weight of Lats $^{-1-}$ mice versus wildtype control mice $(n=4)$. (d) NADH-Tetrazolium Reductase (NADH-TR) staining of

\section{Lats 1 deletion alters muscle fiber type distribution}

Next, we quantified the number and size of type I and IIa muscle fibers in the soleus as it is possible to count all fibers in this muscle. To do so we stained muscle fibers using an ATPase reaction with a $\mathrm{pH} 4.47$ preincubation which stains type I fibers black and type IIa fibers white/grey (Fig. 2a). This revealed that Lat$s 1^{-/-}$mice had $11 \%$ more type I fibers and $11 \%$ fewer type IIa fibers than age and sex-matched wild-type controls $(p<0.05$; Fig. $2 \mathrm{~b}-\mathrm{c})$. In contrast, the muscles of other Hippo-pathway-mutated mice did not differ from matched wild-type controls (Fig. 2b-c and Fig. S2). Consistent with this, in soleus the concentration of slow type I myosin heavy chain (MyHC-1) was $80 \%$ higher in Lats $^{-1-}$ mice than in wildtype control mice $(P<0.05)$ (Fig. 2d). Also, Myh7 mRNA was $50 \%$ higher $(P=0.067)$ in the soleus of Lats $1^{-l-}$ mice than in wild-type controls (Fig. 2e).

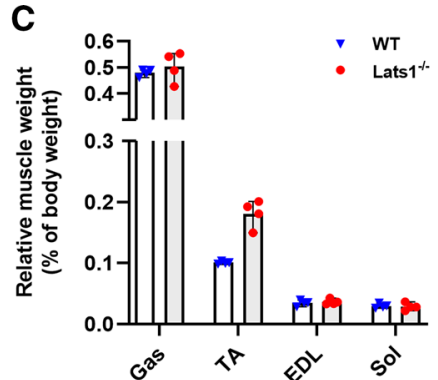

E Soleus muscle

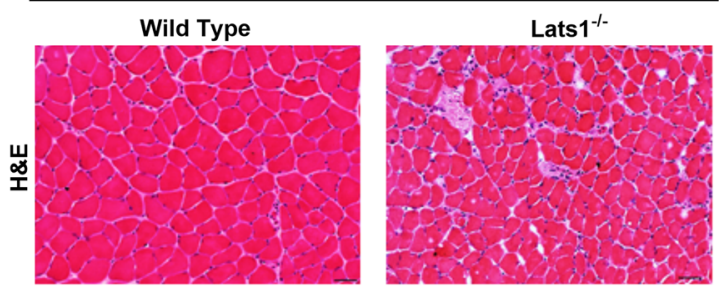

tibialis anterior muscle cross-sections from control and Lats $^{-/-}$ mice (high oxidative capacity is stained in dark blue, low oxidative capacity is stained in light blue; $n=4$ ). (e) Hematoxylin and Eosin (H\&E) staining of the soleus muscle crosssections from control and Lats $1^{-1-}$ mice $(n=4)$. Scale Bar $=50 \mu \mathrm{m}$. All values are presened as mean \pm SEM. ${ }^{*} P<0.05$. KO: Knock Out; WT: Wild Type; g: gram; mg: milligram. See Figure S1 for further H\&E and NADH staining

Effect of exercise-mimicking compounds on Lats1 expression in vitro

To investigate whether Lats1 is regulated by stimuli associated with exercise, we incubated $\mathrm{C} 2 \mathrm{C} 12$ myotubes with the energy stress-inducing AMPKactivating drug AICAR and the hypertrophy-inducing $\beta 2$-agonist Clenbuterol. We then measured Lats1 gene expression (Fig. 3a-b).

After $24 \mathrm{~h}$ treatment with $100 \mu \mathrm{M}$ of clenbuterol, the expression levels of Lats1 increased 1.2 fold (Fig. 3a) when compared to control. After $24 \mathrm{~h}$ of treatment with $1 \mathrm{mM}$ of AICAR Lats1 gene expression increased 1.3 fold when compared to control (Fig. 3b) suggesting that exercise-related stimuli modulate Lats1 expression. 
A
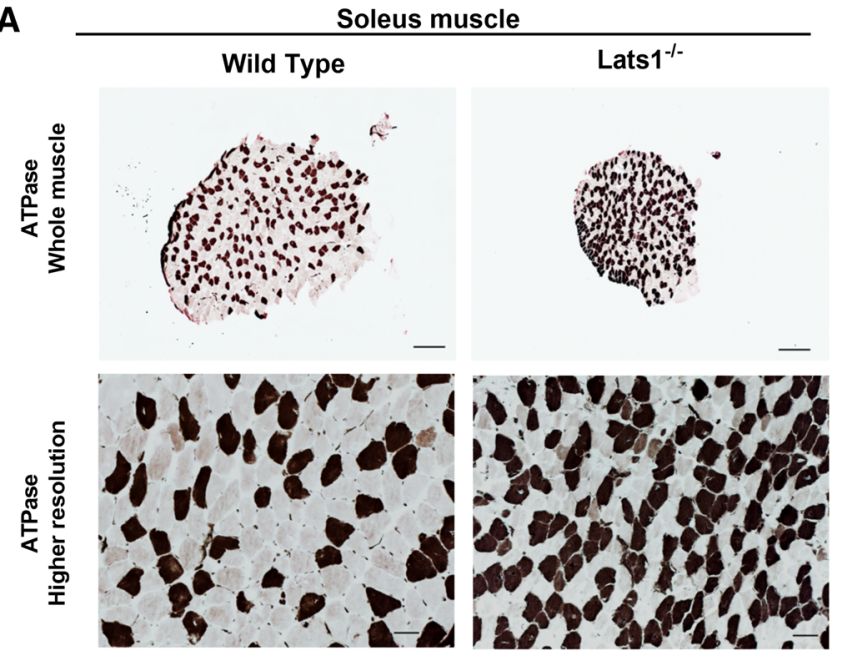

D

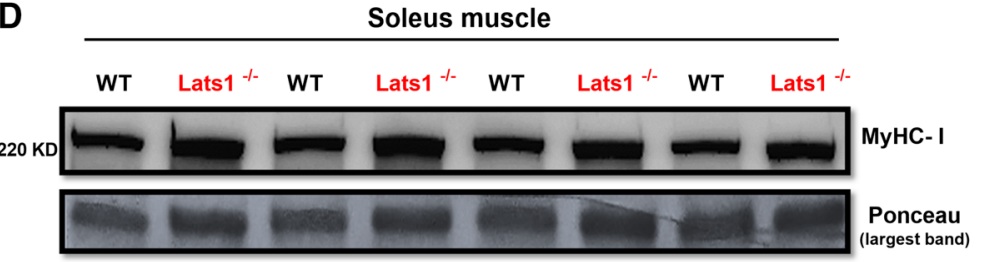

B

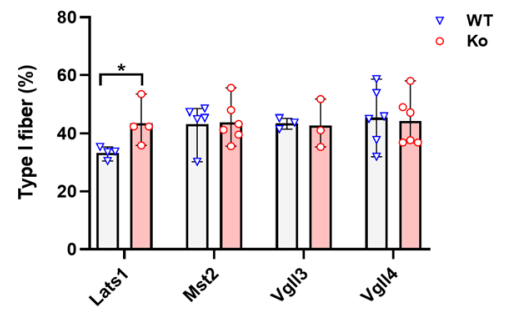

C

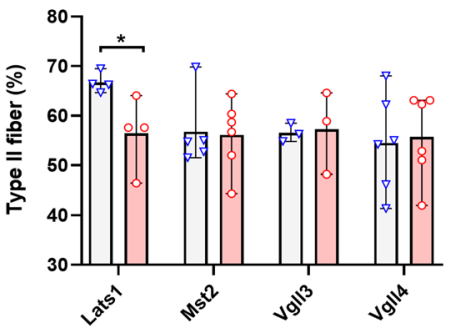

E

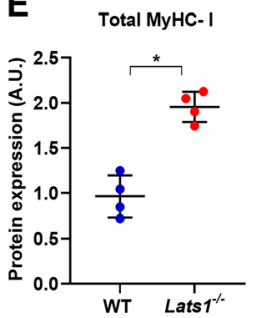

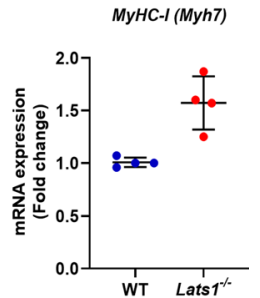

Fig. 2 Lats 1 deletion increases slow type I MyHC. (a) Representative images of ATPase stained soleus muscles crosssections from 16-week-old control and Lats $^{-1-}$ mice $(\mathrm{n}=4)$ to determine fiber types (Type I fibers stain dark, type II and IIa fibers stain light). (b) Type I and (c) IIa fibre percentages in soleus muscles $(\mathrm{n}=3-6)$. (d) Protein levels of total MyHC-I in the soleus muscle from control and Lats ${ }^{-1-}$ mice $(\mathrm{n}=4)$. (e) Expression levels of $M y h$ mRNA (encoding MyHC-I

Bioinformatic analyses of Lats1 gene expression in skeletal muscle

To better understand the regulation of Lats1 expression and phosphorylation in skeletal muscle we retrieved published datasets from Gene Omnibus or downloaded supplemental data from published papers. This revealed that Lats1 is more expressed in slow type I muscle fibers in mice than in fast type II fibers (Chemello et al. 2011). The reanalyzed data from Haslett et al. (see Supplementary Data S1A in Haslett et al. 2003) also revealed that mean LATS1 expression is $63 \%$ higher $(P=0.014)$ in the quadriceps of boys with Duchenne Muscular Dystrophy (DMD) when compared to normal quadriceps muscle (Haslett et al. protein) in the soleus muscles from control and Lats $1^{-1-}$ mice were measured by qPCR $(n=4)$. Protein is normalized to the largest band in the Ponceau stain. Rpl7 was used as a reference gene to normalize mRNA. Circles indicate individual data points. A.U, arbitrary units; KD, Kilo Dalton. Scale Bar $=200 \mu \mathrm{m}$ (whole muscle); $50 \mu \mathrm{m}$ (higher resolution). All values present mean \pm SEM. $* P<0.05$. See Fig. S2 for further ATPase staining figures

2003) (Fig. 4a). Moreover, Lats 1 expression increases in regenerating tibialis anterior muscles by $17-77 \%$ (Lukjanenko et al. 2013) and in hypertrophying plantaris muscle by $41-71 \%$ (See the microarray dataset GSE47098 data (Chaillou et al. 2013)) (Fig. $4 \mathrm{~b}-\mathrm{c}$ ), suggesting that Lats 1 is transcriptionally regulated in muscle. In human vastus lateralis, mean LATS 1 expression declines by $19 \%$ at $2.5 \mathrm{~h}$ and $21 \%$ at $5 \mathrm{~h}(21 \%)$ after endurance exercise (Fig. 4d) (Vissing and Schjerling 2014). However, a MetaMex analysis (Pillon et al. 2020) revealed no significant expression changes of LATS1 in different human skeletal muscles after acute or chronic endurance or resistance exercise (Fig. 4e-f), respectively. 


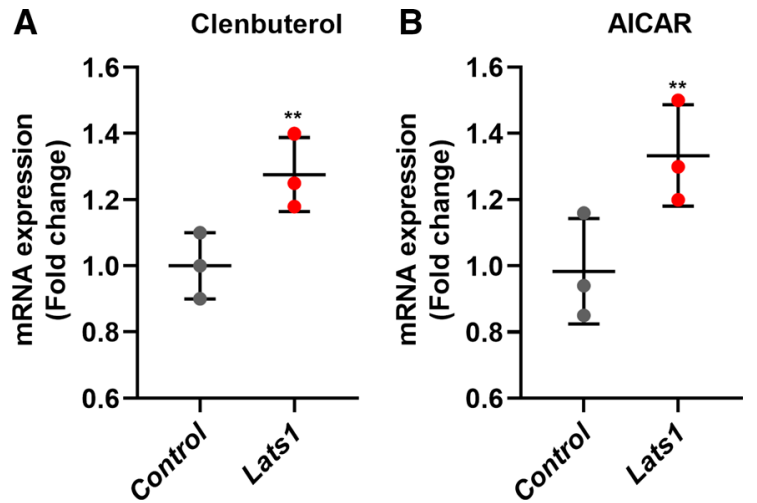

Fig. 3 Effect of exercise-associated stimuli on the expression level of the Lats1 gene in vitro. C2C12 myotubes were incubated with (a) Clenbuterol $(100 \mu \mathrm{M})$, or (b) AICAR $(1 \mathrm{mM})$ for $24 \mathrm{~h}$ and analyzed for Lats 1 gene expression by qRT-PCR. Rpl7 was used as a reference gene to normalize mRNA. The circles indicate individual data points. Data are presented as mean \pm SEM. $* * P<0.001$

\section{Discussion}

In this study, we compared the hindlimb muscles of Lats ${ }^{-/-}$, Mst $^{-/-}$, Vgll $^{-/-}$, and $\mathrm{Vgll4}^{+/-}$transgenic mice with muscles of sex and age-matched control mice. The major finding of this analysis is that deleting Lats $^{-/-}$had $11 \%$ more type I fibers, $50 \%$ more Myh7 mRNA, and 80\% more type I myosin heavy chain when compared to matched wild-type controls. This suggests that Lats1 regulates fiber type proportion in mice. Furthermore, Lats 1 gene expression is upregulated in $\mathrm{C} 2 \mathrm{C} 12$ myotubes treated with either the hypertrophy-stimulating agent clenbuterol or the energy stress-related drug AICAR. Moreover, re-analysis of published datasets revealed that Lats 1 expression increases after muscle injury and synergist ablation and that LATS1 expression is higher in the muscles of young boys with DMD than in healthy muscles.

Lats1 deletion contributes to regulating muscle fiber type distribution in mouse skeletal muscle

The finding that Lats 1 deletion increased the proportion of slow, type I fibers fits the earlier discovery that a loss of $\mathrm{Vgll2}$ in mice reduced type I fibers when compared to wild-type mice (Honda et al. 2017). The fact that Lats1 is an inhibitor of Yap/Taz-Tead1-4 and that Vgll2 is a Tead1-4 coregulator suggest that a change of Tead1-4 activity in muscle can shift the fiber type composition of skeletal muscle. This adds the Hippo pathway to the list of pathways that regulate fiber type proportions. Other pathways in this list are the calcineurin-NFAT and ERK pathways (Ehlers et al. 2014; Schiaffino and Reggiani 2011). However, other Hippo gene mutations did not affect the distribution of muscle fiber types and it is unclear why some Hippo genes can have this effect whilst others have not.

Regulation of Lats1 expression in skeletal muscle

We then performed $\mathrm{C} 2 \mathrm{C} 12$ cell culture experiments and reanalyzed published data sets to find out whether Lats 1 expression or phosphorylation is affected by exercise, damage, disease, or specific drugs. First, we experimentally investigated whether Lats 1 expression is changed in $\mathrm{C} 2 \mathrm{C} 12$ myotubes hypertrophy-stimulated with clenbuterol or exposed to energy stress via AICAR. We found that AICAR increases Lats 1 expression by 1.3 fold. Up-regulation of Lats 1 in response to energy stress treatment with AICAR has been reported in different cell models such as in mouse embryonic fibroblasts and human retinal pigment epithelial-1 cells (Philippe et al. 2018). This suggests that Lats1 expression responds to exercise-related stimuli that may affect muscle fiber type-specific gene expression. Second, our re-analysis of existing datasets revealed that Lats 1 expression is elevated in synergist ablation-loaded hypertrophying plantaris muscle (Lukjanenko et al. 2013). Additionally, Lats 1 expression is upregulated in regenerating muscles after cardiotoxin-induced injury (Chaillou et al. 2013) (Fig. 4 b-c). The reanalysis of published datasets also revealed that LATS1 expression is $63 \%$ higher in the quadriceps of young boys with Duchenne muscular dystrophy compared to healthy quadriceps muscle (Fig. 4 a). Vita et al. 2018 also reported that LATS1/2 kinase activity increases in five different muscles (quadriceps, biceps, diaphragm, gastrocnemius, and EDL) in mdx mice that is a popular model for studying DMD. In DMD muscles, high miR-21 expression leads to an increase of Lats activity. Activation of both miR-21 and Lats 1 can cause direct suppression of Yap activity and results in muscle degeneration and weakness (Vita et al. 2018). These data suggest that Lats1 is transcriptionally regulated in skeletal muscle.

The limitation of the study is that we only compared 3-5 mutant hindlimbs (Lats $1^{-/-}(\mathrm{n}=4)$, Mst $^{-/-}$ 
A

LATS1

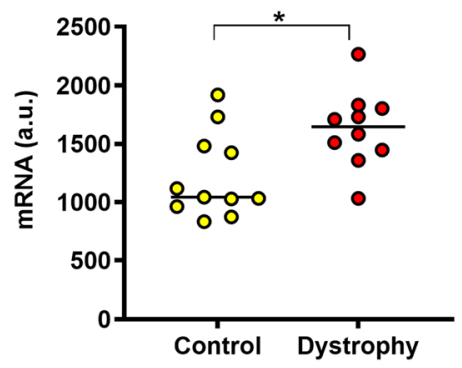

C

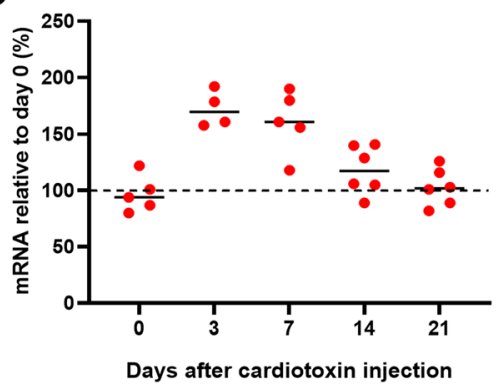

E

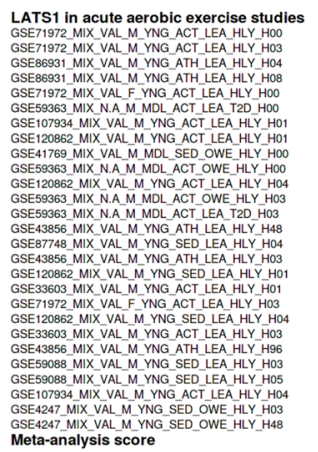

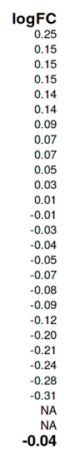

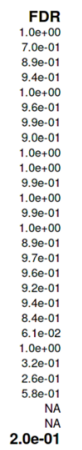

$n$
8
8
2
2
2
4
7
6
7
9
7
7
7
7
16
10
16
7
11
4
7
11
16
6
6
6

197
197

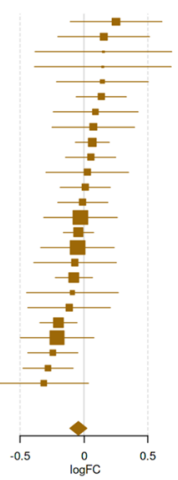

Fig. 4 Lats1 gene expression in response to physiological and pathological factors in skeletal muscle. (a) LATS1 expression in quadriceps muscle biopsy samples of healthy and DMD patients (Haslett et al., 2003). (b) Relative mRNA expression of Lats1 in synergist ablation-overloaded mouse plantaris muscle (Chaillou et al 2013). (c) Latsl gene expression in mice tibialis anterior muscle injured with cardiotoxin injection at day 0 up to day 21 (Lukjanenko et al. 2013). (d) Effect of human endurance and resistance (strength) exercise on the expression of LATS1 in the

$(\mathrm{n}=5), \operatorname{Vgll}^{-1-}(\mathrm{n}=3)$, and $\operatorname{Vgll}^{+/-}(\mathrm{n}=5)$ to age and sex-matched wild-type controls. However, we additionally measured $M y h 7 \mathrm{mRNA}$ and myosin type I

B

Lats1

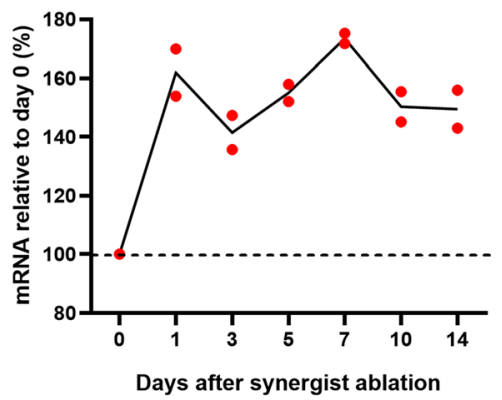

D

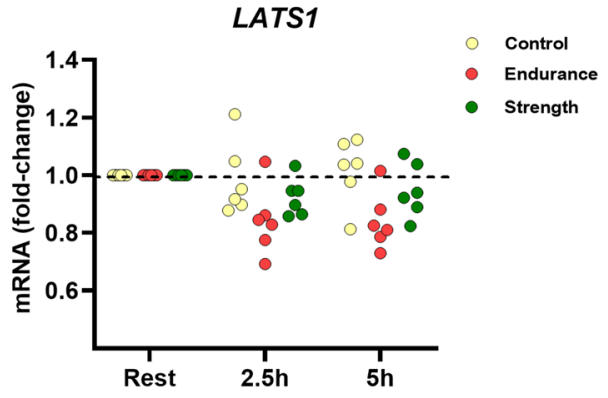

$\mathbf{F}$
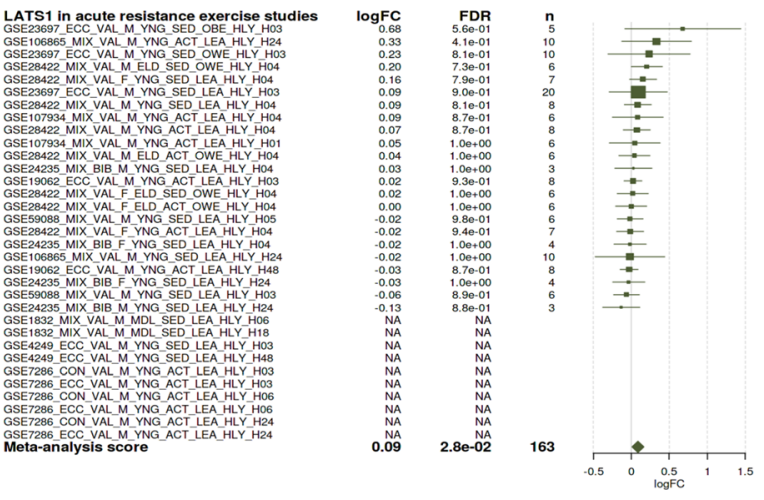

vastus lateralis $2.5 \mathrm{~h}$ and $5 \mathrm{~h}$ after exercise (Vissing and Schjerling, 2014). The circles indicate individual data points. (e-f) Meta-analysis of Lats1 expression in response to (e) acute aerobic and (f) acute resistance exercise (http://www.metamex. eu/) (Pillon et al., 2020). Muscle Biopsies were collected at $0 \mathrm{~h}$ up to $96 \mathrm{~h}$ after exercise. The fold-change $(\log 2)$ is represented by a square and the $95 \%$ confidence intervals are represented by horizontal lines. LogFC, Fold-change $(\log 2)$; FDR, false discovery rate; n, sample size; A.U, arbitrary units. ${ }^{*} P<0.05$

protein levels to confirm ourselves of the shift in fiber type.

In summary, Lats $^{-/-}$mice had $11 \%$ more type I fibers, and that Lats $^{-/-}, \mathrm{Mst}^{-/-}, \mathrm{Vgll}^{-/-}, \mathrm{Vgll4}^{+/-}$ 
transgenic mice have no pathological skeletal muscle phenotype. Moreover, synergist ablation, muscle injury, and muscular dystrophy all increase the expression of Lats1.

Acknowledgements We thank the MRC Harwell facility for generating and providing muscle samples of Lats $^{-/}, \mathrm{Mst}^{-{ }^{-/}}$and $\mathrm{Vgll}^{-/}$mice as well as sex-and age-matched controls. We also thank the German Mouse Clinic for generating and providing $\mathrm{Vgll}^{+/-}$mice and sex- and age-matched controls.

Authors' contribution Conceptualization by HW, MS, LB \& $\mathrm{MH}$; methodology AR, FYN; formal analysis FYN, AR, MS, HW; data curation AR, FYN, MS; writing original draft preparation, HW, FYN; writing review and editing by HW, FYN, MS, LB, MH. All authors have read and approved the final version of the manuscript.

Funding Open Access funding enabled and organized by Projekt DEAL. This work was supported by grants from the German Federal Ministry of Education and Research (Infrafrontier grant 01KX1012 to MHdA), and German Center for Diabetes Research (DZD) (MHdA).

\section{Declarations}

Conflict of interest The authors declare no conflicts of interest.

Ethical approval All animal procedures include ethical statements condition that is available on the IMPC portal (http:// www.mousephenotype.org/about-impc/arrive-guidelines).

Consent to participate Not applicable.

Consent for publication Not applicable.

Open Access This article is licensed under a Creative Commons Attribution 4.0 International License, which permits use, sharing, adaptation, distribution and reproduction in any medium or format, as long as you give appropriate credit to the original author(s) and the source, provide a link to the Creative Commons licence, and indicate if changes were made. The images or other third party material in this article are included in the article's Creative Commons licence, unless indicated otherwise in a credit line to the material. If material is not included in the article's Creative Commons licence and your intended use is not permitted by statutory regulation or exceeds the permitted use, you will need to obtain permission directly from the copyright holder. To view a copy of this licence, visit http://creativecommons.org/licenses/by/4.0/.

\section{References}

Bonaldo P, Sandri M (2013) Cellular and molecular mechanisms of muscle atrophy. Dis Model Mech 6(1):25-39. https://doi.org/10.1242/dmm.010389

Chaillou T, Lee JD, England JH, Esser KA, McCarthy JJ (2013) Time course of gene expression during mouse skeletal muscle hypertrophy. J Appl Physiol 115(7):1065-1074. https://doi.org/10.1152/japplphysiol.00611.2013

Chemello F, Bean C, Cancellara P, Laveder P, Reggiani C, Lanfranchi G (2011) Microgenomic analysis in skeletal muscle: expression signatures of individual fast and slow myofibers. PLoS ONE 6(2):e16807. https://doi.org/10. 1371/journal.pone.0016807

Dong J, Feldmann G, Huang J, Wu S, Zhang N, Comerford SA, Gayyed MF, Anders RA, Maitra A, Pan D (2007) Elucidation of a universal size-control mechanism in Drosophila and mammals. Cell 130(6):1120-1133. https://doi.org/10. 1016/j.cell.2007.07.019

Ehlers ML, Celona B, Black BL (2014) NFATc1 controls skeletal muscle fiber type and is a negative regulator of MyoD activity. Cell Rep 8(6):1639-1648. https://doi.org/ 10.1016/j.celrep.2014.08.035

Figeac N, Mohamed AD, Sun C, Schönfelder M, Matallanas D, Garcia-Munoz A, Missiaglia E, Collie-Duguid E, De Mello V, Pobbati AV, Pruller J, Jaka O, Harridge SDR, Hong W, Shipley J, Vargesson N, Zammit PS, Wackerhage H (2019) VGLL3 operates via TEAD1, TEAD3 and TEAD4 to influence myogenesis in skeletal muscle. J Cell Sci. https:// doi.org/10.1242/jcs. 225946

Gabriel BM, Hamilton DL, Tremblay AM, Wackerhage H (2016) The Hippo signal transduction network for exercise physiologists. J Appl Physiol 120(10):1105-1117. https:// doi.org/10.1152/japplphysiol.01076.2015

Honda M, Hidaka K, Fukada S, Sugawa R, Shirai M, Ikawa M, Morisaki T (2017) Vestigial-like 2 contributes to normal muscle fiber type distribution in mice. Sci Rep 7:7168

Haslett JN, Sanoudou D, Kho AT, Han M, Bennett RR, Kohane IS, Beggs AH, Kunkel LM (2003) Gene expression profiling of Duchenne muscular dystrophy skeletal muscle. Neurogenetics 4(4): 163-171. <Go to ISI >:// MEDLINE: 12698323

Hori N, Okada K, Takakura Y, Takano H, Yamaguchi N, Yamaguchi N (2020) Vestigial-like family member 3 (VGLL3), a cofactor for TEAD transcription factors, promotes cancer cell proliferation by activating the Hippo pathway. J Biol Chem 295(26):8798-8807. https://doi.org/ 10.1074/jbc.RA120.012781

Janssen I, Heymsfield SB, Wang ZM, Ross R (2000) Skeletal muscle mass and distribution in 468 men and women aged 18-88 yr. J Appl Physiol 89(1):81-88. https://doi.org/10. 1152/jappl.2000.89.1.81

Koontz LM, Liu-Chittenden Y, Yin F, Zheng Y, Yu J, Huang B, Chen Q, Wu S, Pan D (2013) The Hippo effector Yorkie controls normal tissue growth by antagonizing scallopedmediated default repression. Dev Cell 25(4):388-401. https://doi.org/10.1016/j.devcel.2013.04.021

Lexell J, Taylor CC, Sjöström M (1988) What is the cause of the ageing atrophy? Total number, size and proportion of different fiber types studied in whole vastus lateralis muscle 
from 15- to 83-year-old men. J Neurol Sci 84(2-3):275-294. https://doi.org/10.1016/0022$510 x(88) 90132-3$

Liang N, Zhang C, Dill P, Panasyuk G, Pion D, Koka V, Gallazzini M, Olson EN, Lam H, Henske EP, Dong Z, Apte U, Pallet N, Johnson RL, Terzi F, Kwiatkowski DJ, Scoazec JY, Martignoni G, Pende M (2014) Regulation of YAP by mTOR and autophagy reveals a therapeutic target of tuberous sclerosis complex. J Exp Med 211(11):2249-2263. https://doi.org/10.1084/jem. 20140341

Liu CY, Zha ZY, Zhou X, Zhang H, Huang W, Zhao D, Li T, Chan SW, Lim CJ, Hong W, Zhao S, Xiong Y, Lei QY, Guan KL (2010) The hippo tumor pathway promotes TAZ degradation by phosphorylating a phosphodegron and recruiting the SCF\{beta\}-TrCP E3 ligase. J Biol Chem 285(48):37159-37169. https://doi.org/10.1074/jbc.M110. 152942

Livak KJ, Schmittgen TD (2001) Analysis of relative gene expression data using real-time quantitative PCR and the 2(-Delta Delta C(T)) Method. Methods 25(4):402-408. https://doi.org/10.1006/meth.2001.1262

Lukjanenko L, Brachat S, Pierrel E, Lach-Trifilieff E, Feige JN (2013) Genomic profiling reveals that transient adipogenic activation is a hallmark of mouse models of skeletal muscle regeneration. PLoS ONE 8(8):e71084. https://doi.org/10. 1371/journal.pone.0071084

Oh H, Irvine KD (2008) In vivo regulation of Yorkie phosphorylation and localization. Development 135(6):1081-1088. https://doi.org/10.1242/dev.015255

Philippe C, Pinson B, Dompierre J, Pantesco V, Viollet B, Daignan-Fornier B, Moenner M (2018) AICAR antiproliferative properties involve the AMPK-independent activation of the tumor suppressors LATS 1 and 2. Neoplasia 20(6):555-562. https://doi.org/10.1016/j.neo.2018.03.006

Pillon NJ, Gabriel BM, Dollet L, Smith JAB, Sardón Puig L, Botella J, Bishop DJ, Krook A, Zierath JR (2020, 2020/01/ 24) Transcriptomic profiling of skeletal muscle adaptations to exercise and inactivity. Nat Commun 11(1): 470. https:// doi.org/10.1038/s41467-019-13869-w

Ren F, Zhang L, Jiang J (2010) Hippo signaling regulates Yorkie nuclear localization and activity through 14-3-3 dependent and independent mechanisms. Dev Biol 337(2):303-312. https://doi.org/10.1016/j.ydbio.2009.10. 046

Romero-Calvo I, Ocón B, Martínez-Moya P, Suárez MD, Zarzuelo A, Martínez-Augustin O, de Medina FS (2010) Reversible Ponceau staining as a loading control alternative to actin in Western blots. Anal Biochem 401(2):318-320. https://doi.org/10.1016/j.ab.2010.02.036

Sarzynski MA, Bouchard C (2020) World-class athletic performance and genetic endowment. Nat Metab 2(9):796-798. https://doi.org/10.1038/s42255-020-0233-6
Schiaffino S, Reggiani C (2011) Fiber types in mammalian skeletal muscles. Physiol Rev 91(4):1447-1531. https:// doi.org/10.1152/physrev.00031.2010

St John MA, Tao W, Fei X, Fukumoto R, Carcangiu ML, Brownstein DG, Parlow AF, McGrath J, Xu T (1999) Mice deficient of Lats1 develop soft-tissue sarcomas, ovarian tumours and pituitary dysfunction. Nat Genet 21(2):182-186. https://doi.org/10.1038/5965

Thomas KC, Zheng XF, Garces Suarez F, Raftery JM, Quinlan KG, Yang N, North KN, Houweling PJ (2014) Evidence based selection of commonly used RT-qPCR reference genes for the analysis of mouse skeletal muscle. PLOS ONE 9(2):e88653. https://doi.org/10.1371/journal.pone. 0088653

Vissing K, Schjerling P (2014) Simplified data access on human skeletal muscle transcriptome responses to differentiated exercise. Sci Data 1:140041. https://doi.org/10.1038/sdata. 2014.41

Vita GL, Polito F, Oteri R, Arrigo R, Ciranni AM, Musumeci O, Messina S, Rodolico C, Di Giorgio RM, Vita G, Aguennouz M (2018) Hippo signaling pathway is altered in Duchenne muscular dystrophy. PLoS ONE 13(10):e0205514. https://doi.org/10.1371/journal.pone. 0205514

Wackerhage H, Del Re DP, Judson RN, Sudol M, Sadoshima J (2014) The Hippo signal transduction network in skeletal and cardiac muscle. Sci Signal 7(337):re4. https://doi.org/ 10.1126/scisignal.2005096

Wolfe RR (2006) The underappreciated role of muscle in health and disease. Am J Clin Nutr 84(3):475-482. https://doi. org/10.1093/ajcn/84.3.475

Yamaguchi N (2020) Multiple roles of vestigial-like family members in tumor development. Front Oncol 10:1266. https://doi.org/10.3389/fonc.2020.01266

Zhao B, Li L, Tumaneng K, Wang CY, Guan KL (2010) A coordinated phosphorylation by Lats and CK1 regulates YAP stability through SCF(beta-TRCP). Genes Dev 24(1):72-85. https://doi.org/10.1101/gad.1843810

Zhao J, Brault JJ, Schild A, Cao P, Sandri M, Schiaffino S, Lecker SH, Goldberg AL (2007) FoxO3 coordinately activates protein degradation by the autophagic/lysosomal and proteasomal pathways in atrophying muscle cells. Cell Metab 6(6):472-483. https://doi.org/10.1016/j.cmet.2007. 11.004

Zhou Y, Huang T, Cheng AS, Yu J, Kang W, To KF (2016) The TEAD family and its oncogenic role in promoting tumorigenesis. Int J Mol Sci. https://doi.org/10.3390/ ijms 17010138

Publisher's Note Springer Nature remains neutral with regard to jurisdictional claims in published maps and institutional affiliations. 